\title{
STEPSIM4 Revised: Network Analysis Methodology for Critical Paths in Rock Mass Slopes
}

\author{
N.R.P. Baczynski Ok Tedi Mining Ltd, Papua New Guinea
}

\begin{abstract}
This paper explains the conceptual framework for a revised network analysis version of STEPSIM4 software that is used for estimating the statistical shear strength developed along critical step-path traverses through jointed rock mass slopes. Limitations of STEPSIM4 are discussed and related to the revised conceptual framework. Network analysis techniques offer a unique opportunity for efficiently searching through a complex rock mass system comprising elements of the rock mass, intact rock and defects to locate the critical minimum shear strength path. Development of the revised network analysis software version, STEPSIM5, is being sponsored and is scheduled for completion in 2008.
\end{abstract}

\section{Introduction}

STEPSIM4 is a Monte Carlo simulation step-path method for determining the statistical shear strength along two-dimensional (2D) critical failure paths through jointed rock slopes (Baczynski, 2000 and Baczynski et al., 2001). This analysis method is suitable for sliding failures but unsuitable for toppling failures.

In this paper, the term 'defect' broadly refers to any type of natural occurring structural geological discontinuity in the rock mass; irrespective of its tectonic origin (i.e. joint, shear, fault, bedding, foliation or rock type contact). This approach has been used because geologists do not always distinguish between defect types during mapping (e.g. bedding and foliation; subhorizontal conjugate shears and subhorizontal joints). A 'set' refers to a group of defects that have statistically similar orientations. Members of a defect 'set' are defined in terms of their mean orientation (i.e. stated in terms of dip direction and dip angle) and the statistical scatter (i.e. standard deviation) of their orientations about this mean.

STEPSIM4 can simulate two defect sets along the step-path traverse through the slope. The mean dip of this traverse depends on three factors, namely, the relative occurrence of each defect set within the rock mass, the statistical variation in dip and length defects in each set and the statistical variation in length of intact rock/rock mass bridges between defects in each set. Traverse shear strength is the cumulative sum of strengths of the elements defining the traverse. The statistical shear strength model for critical step-path traverses is developed by iterative Monte Carlo STEPSIM4 simulation of a large number of traverses (say 2000 to 5000).

The initial step-path software was conceptualised by Dr Barry McMahon in 1979 during his pit slope design work for the Bougainville open pit mine in Papua New Guinea (PNG); resulting in the STPSIM software being coded in the FORTRAN programming language in 1981 to run on mainframe computers. Several minor code modifications were made during the 1980s. STPSIM was transported to the Ok Tedi Mine in PNG in 1991 and adapted to run on personal computers. During the 1997-2000 pit slope design optimisation study for Ok Tedi Mine (Little et al., 1997-2000), the STPSIM software was extensively modified and rewritten as STEPSIM4 by the author.

A number of logic and analysis limitations and assumptions became apparent during the upgrade of the STPSIM software into STEPSIM4. Of necessity, much of the basic structure and flow of the initial STPSIM code was retained in STEPSIM4 but the program's capabilities were streamlined, statistically enhanced and the internal workings of the software code made more transparent to the user. Removal of identified deficiencies would have involved changing the underlying program logic and necessitated a total rewrite of the software. 
The STEPSIM4-REVISED network analysis methodology outlined in this paper resolves the shortcomings in the existing STEPSIM4 approach and provides a better geotechnical tool for computing the shear strength along defect-controlled sliding failure step-path traverses through rock mass slopes.

\section{Existing STEPSIM4 methodology}

\subsection{Defect characteristics}

Rock mass is defined as a system of intact rock and defects. One or more sets of defects often occur. In most rock masses, members of a defect set are rarely uniformly or randomly distributed. As shown in Figure 1, defects tend to occur in clusters (Baczynski, 1980) that may be referred to as zones.

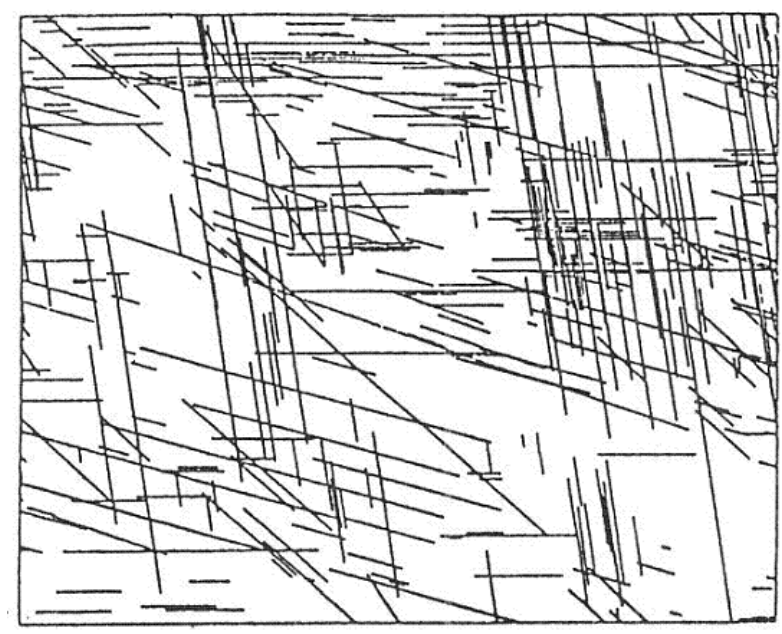

Figure 1 Conceptual rock mass with several defect sets

Geotechnical attributes of each defect set may be statistically characterised.

The key attributes of defects are:

- Orientation.

- Relative proportion of weak defect types, e.g. faults versus joints with similar orientations.

- 'Probability of occurrence' in the rock mass, i.e. presence or absence of members of a particular defect set in 'data windows' used for rock face mapping.

- Length.

- Spacing.

- Large and small-scale defect surface roughness.

- Infill type, thickness and shear strength.

- 'Probability of cut-off' by other defects.

- Length of 'bridges' (i.e. for defects that are not cut-off by other defects). This length is defined as the shortest distance to any other defect in the rock mass.

- Intact rock 'bridge' strength.

- Rock mass 'bridge' strength.

STEPSIM4 considers each attribute in computing shear strength.

Strength variability between successive paths is traverse length dependent. In accordance with statistical sampling theory, the shortest paths display the greatest strength variability. Shear strength along short paths 
may be entirely controlled by a single defect or be devoid of simulated defects (i.e. rock mass strength). Strength along very long paths is the cumulative sum of large numbers of defects and rock bridges.

\subsection{Slope failure modes}

Typical slope failure modes include:

- Sliding on one or more major defects such as faults or master joints (planar, tetrahedral, activepassive wedge failure mechanisms).

- Sliding along quasi-circular failure paths across rock mass structure.

- Toppling.

- Composite modes, involving two or more of the above mechanisms.

Composite modes are common on high slopes.

\subsection{Critical failure path}

Figure 2 shows a conceptual failure traverse through a rock mass slope. A number of aspects are illustrated.

- Conceptually, the critical path is the traverse of least shearing resistance through the slope with respect to the forces activating the instability.

- A single through-going defect such as a fault located at some distance behind the slope face (i.e. this defect does not daylight in the slope face, as shown in the upper third of the slope in Figure 2) may define a major part of the critical path.

- In absence of suitably orientated defects (i.e. as shown in the central third of the slope in Figure 2), the failure path traverses through the rock mass.

- In presence of suitably-orientated short defects (i.e. as shown in the lower third of the slope in Figure 2), the failure path traverses along shallow dipping defects, steps-up on steeper defects and shears through intact rock (or rock mass) bridges between defects. This is important because the vast majority of defects are shorter than typical heights of mine slopes. Defects such as joints are generally shorter than $10 \mathrm{~m}$.

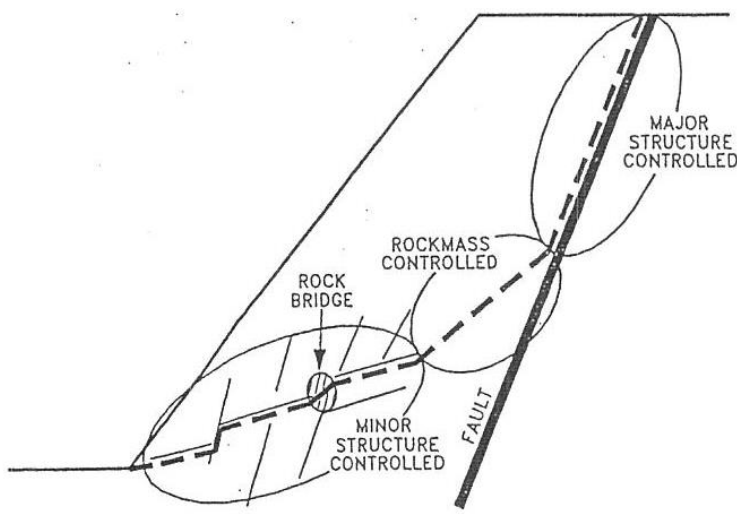

Figure 2 Conceptual slip failure path through a rock mass slope

\subsection{STEPSIM4 approach}

STEPSIM4 conceptualises the traverse through the rock mass slope as comprising a suite of adjacent 'cells'. Dimensions of 'cells' are user specified at the outset of the analysis. 
A maximum of two defect sets (i.e. known as Set 1 and Set 2) may be statistically simulated in each 'cell'; although members of each defect set can be further partitioned by defect type, e.g. major faults and shorter joints, with each type characterised by its own distribution geotechnical attributes. STEPSIM4 assumes that Set 1 and Set 2 defects occur independently within the rock mass (i.e. the defects are not co-dependent).

Four 'cell' conditions are possible.

- Rock mass only (Set 1 and Set 2 defects are absent).

- Set 1 defects and bridges only.

- Set 2 defects and bridges only.

- Both Set 1 and Set 2 defects and bridges.

The STEPSIM4 Monte Carlo statistical simulation process involves the following procedure.

Step 1: The user designates the length of the failure path to be evaluated (e.g. $100 \mathrm{~m}, 400 \mathrm{~m}, 1000 \mathrm{~m}$, and so on). For each simulated failure path, the defect and strength characteristics of each 'cell' are statistically assigned on the basis of the user provided input geotechnical parameter models.

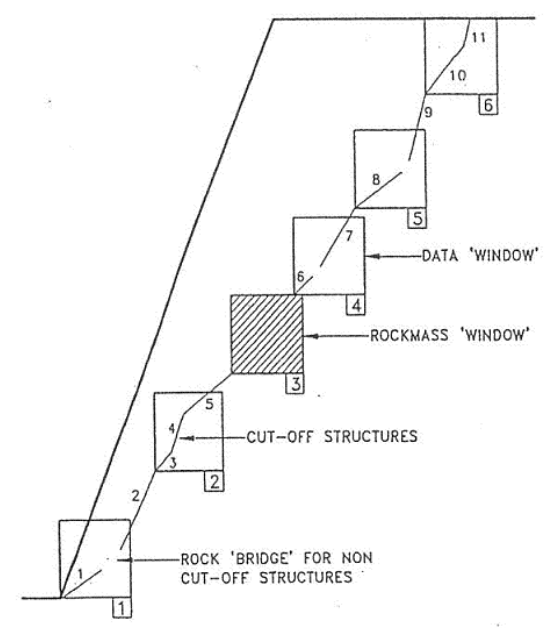

Figure 3 STEPSIM4 conceptual basis and traverse simulation procedure

Step 2: The model slope faces towards the left-hand-side (LHS) and the candidate failure path starts at the toe of this slope and ascends towards the right-hand-side (RHS). The bottom LHS corner of the first ground condition 'cell' coincides with the toe of the model slope. 'Cell' size should be statistically meaningful and, ideally, should reflect the dimensions of the 'data windows' used to structurally map slope faces. If this is not possible, then some arbitrary 'cell' size (say 5 x $5 \mathrm{~m}$ or 10 x $10 \mathrm{~m}$ and so on) may be selected.

Step 3: The statistical model for 'probability of occurrence' of Set 1 and Set 2 defects within the rock mass must be defined. STEPSIM4 then uses a random number generating technique to check whether one, both or neither of the defect sets should be simulated in the first 'cell'. If neither of the sets occurs, then the statistically defined rock mass properties are assigned to the first 'cell'.

Step 4: If one of the sets or both sets occur, then the random number generating Monte Carlo process is again used to systematically generate the respective defects within the first cell. Based on the input statistical model defect type (i.e. fault or joint) for the respective sets, a 'type' is Monte Carlo assigned to the first structure. A similar process is used to assign orientation (dip), length and shear strength to the first defect to check whether the defect terminates in rock or is 'cut-off' by another defect. If the first defect is not 'cut-off', then a statistically assigned length of rock 'bridge', with statistically assigned strength, is simulated at the end of the first defect. The second defect starts at the end of this rock bridge. Depending on their length, bridges may have either intact rock or rock mass shear strength properties assigned by Monte Carlo 
simulation from the respective input statistical distributions for these geotechnical parameters. If both Set 1 and Set 2 occur in the first cell, then the Monte Carlo process is used to decide whether the next structure to be generated should be a Set 1 or a Set 2 member. This STEPSIM 4 process is iterated until the last-generated defect or bridge terminates at the perimeter of the current cell or just outside the perimeter of this cell. This completes simulation for the first cell.

Step 5: The bottom LHS corner of second cell starts at the end of the last-generated defect or bridge. The above simulation process is repeated for the second cell.

Step 6: The above process is repeated for successive cells until the target failure path length has been simulated and the respective shear strength parameters and large-scale roughness are computed.

Step 7: The STEPSIM4 process is repeated for a large number (usually 2000 to 5000) of traverses and the ensuing statistical distribution of shear strength is computed (i.e. the mean and standard deviation with respect to effective friction angle and cohesion).

The STEPSIM4 derived shear strength parameters provide the basic input to slope stability analyses. These analyses may be undertaken by means of either conventional deterministic or Monte Carlo based probabilistic stability analysis software packages.

\section{Assumptions and limitations in STEPSIM4}

A number of conceptual assumptions and limitations exist in STEPSIM4.

The assumptions are:

- The average dip of a traverse, and direction of sliding within the rock mass, coincide with the straight line that connects the start of the traverse at the slope toe with the end of the traverse where it exits at some location behind the slope crest (as shown in Figures 4 and 5).

- Sliding type failure mode occurs on all defects set orientations.

- Sliding and step-ups occur along the entire defect lengths (as shown in Figure 5).
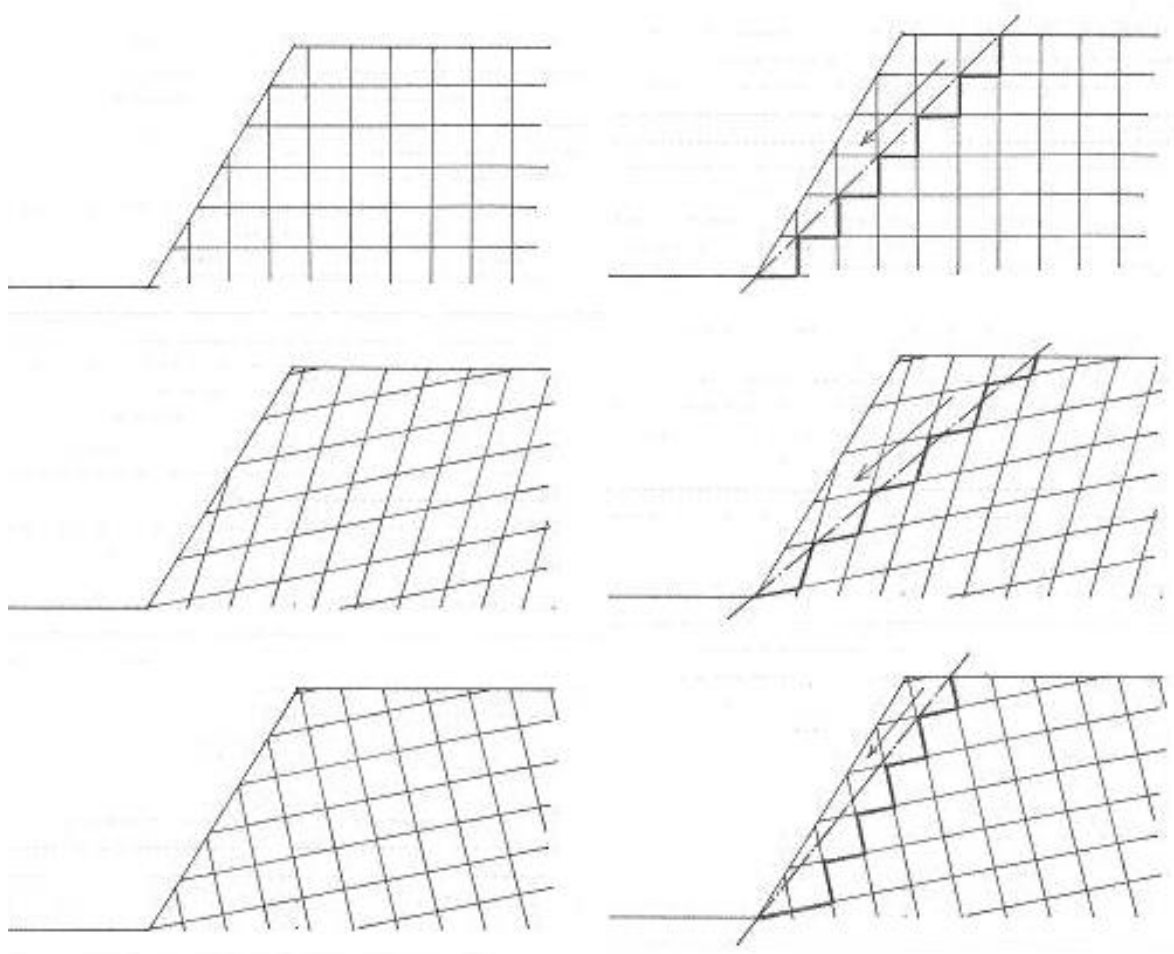

Figure 4 STEPSIM4 mean paths and sliding directions (100\% continuous defects) 
These assumptions may only be correct in some instances. Even when rock masses comprise $100 \%$ continuous defects (as shown in Figure 6), the more likely ground behaviour will be:

- Sliding on the shallower dipping defect set.

- Tensile separation on the steeper defect set.

- Direction of sliding parallel to the shallower dipping defect set.
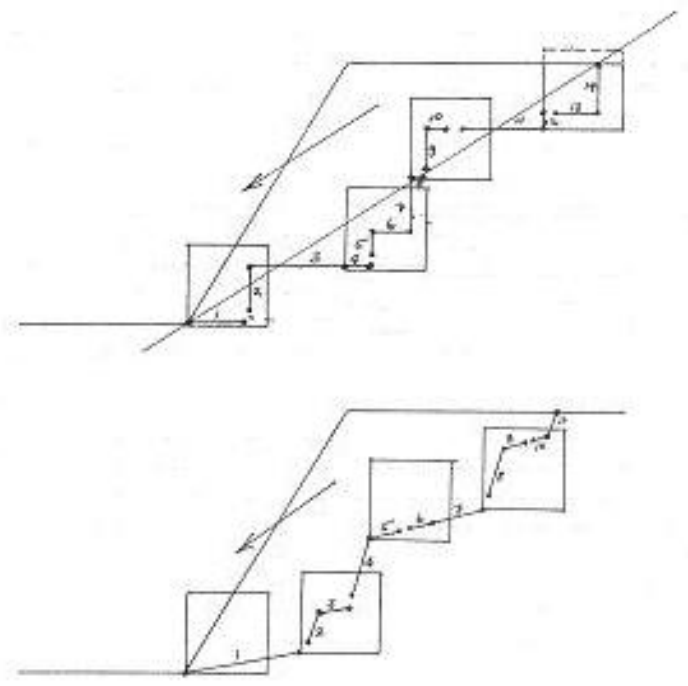

Figure 5 STEPSIM4 mean paths and sliding directions (discontinuous defects)
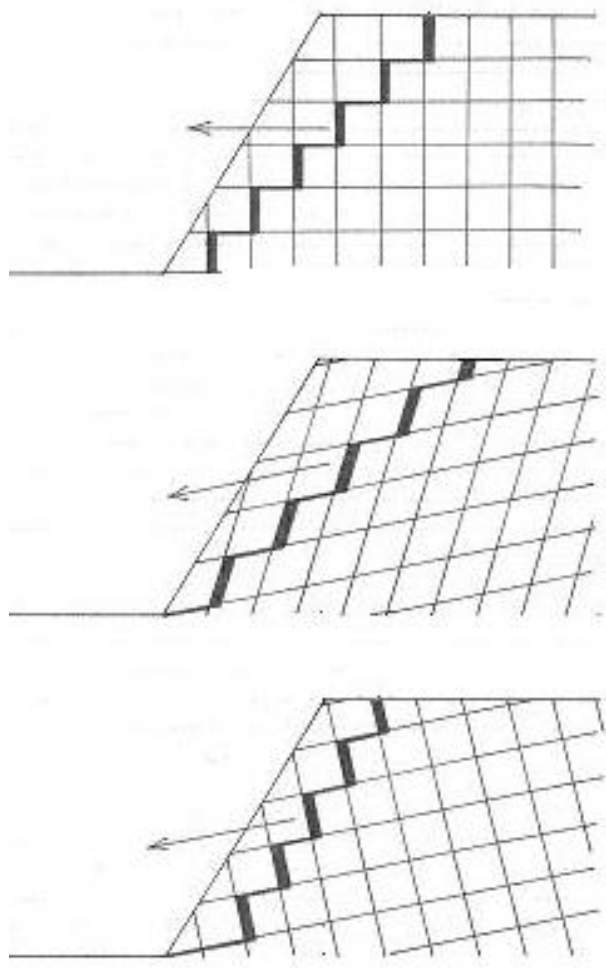

Figure 6 Likely paths, step-ups and sliding directions (100\% continuous defects) 
Defects are rarely evenly spaced and $100 \%$ continuous in real rock masses; the situation is often more complex. As shown in Figure 7, defect attributes and slope failure mechanisms in real rock masses are:

- Members of a defect set that are discontinuous, with a statistical distribution of lengths.

- Members of a defect set that are variously spaced, often occurring in zones or clusters.

- Direction of sliding within the rock slope is parallel to the shallower dipping defect set, at least in the initial stages of failure before the rock mass has significantly dilated.

- Driven by critical failure paths need not follow entire lengths of individual defects, often step-ups on partial defect lengths are common.
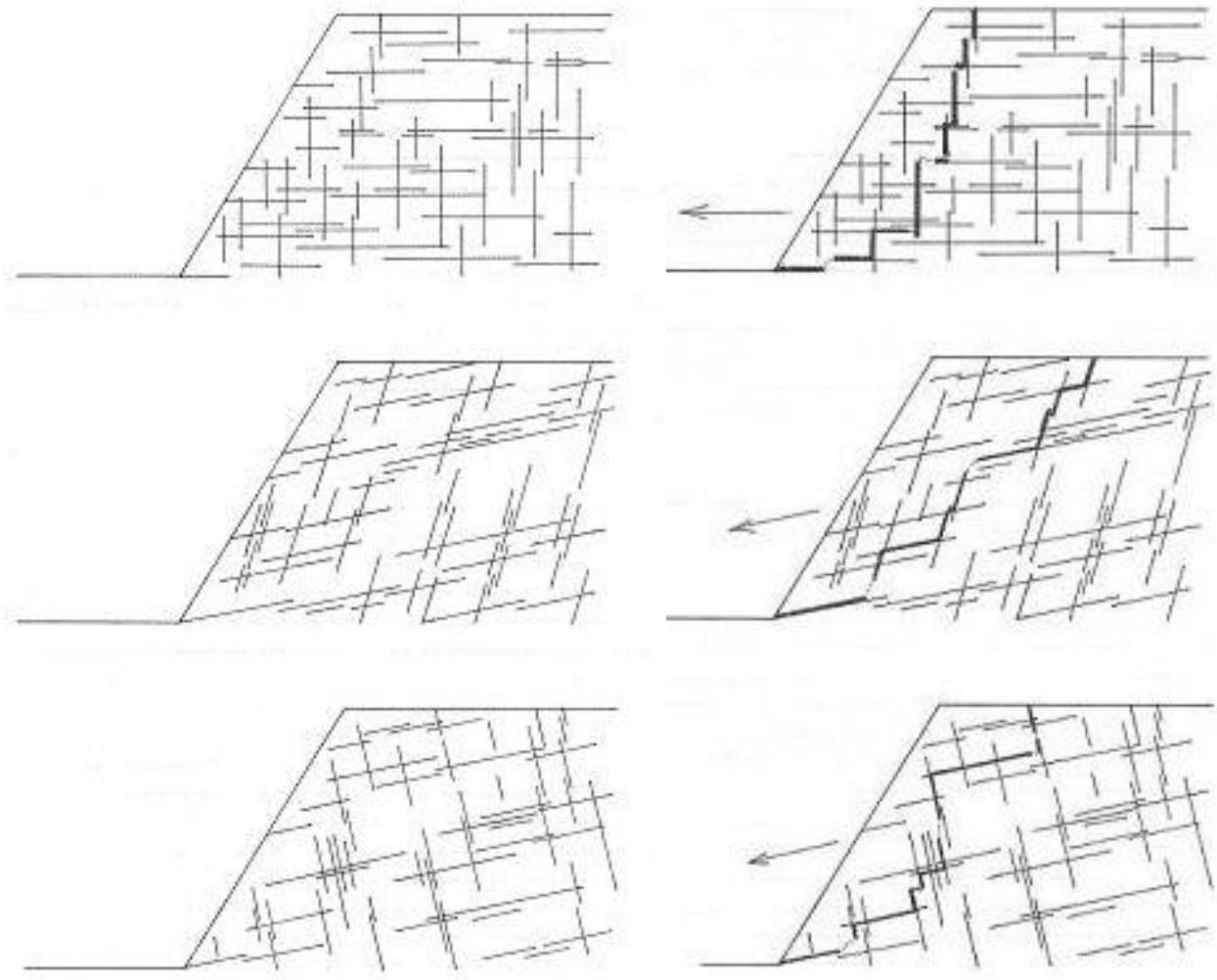

Figure 7 Likely paths, step-ups and sliding directions (discontinuous defects)

The existing STEPSIM4, in summary, uses the Monte Carlo method to statistically simulate defectcontrolled, step-path traverses through rock mass slopes and estimates the effective shear strength along these traverses. Whilst the STEPSIM4 concept is simple, there are several inherent assumptions and limitations in this method that are at variance with actual landslides. The contentious issues are whether the mean dip of a traverse is geotechnically relevant at the onset of instability, whether the mean dip traverse necessarily always coincides with actual direction of sliding, the inability to simulate traverses where at least some step-ups involve only partial rather than full defect lengths, and the inability to simulate tensile separation along steep dipping defect sets rather than assume that sliding failure occurs on all defect sets along the traverse.

\section{Network analysis modelling of step-path traverses}

\subsection{Concept}

The concept of 'network analysis' appears to mean different things to different people (Brandes and Erleback, 2005). Network refers to the informal concept describing an object composed of elements and 
interactions or connections between these elements. Network analysis is carried out in areas such as project planning, complex systems, electrical circuits, social networks, transportation systems, communications networks, epidemiology, bioinformatics, hypertext systems, text analysis, organisation theory, genealogical research and analysis of complex events; just to name a few examples.

Common links in all of the above examples are that the 'problem' or enquiry often contains a vast number of possible 'solutions' or answers. There are three steps in finding the optimum solution.

Step 1: Criteria need to be developed to define 'optimum solution'.

Step 2: Each of the vast number of possible outcomes, which may be considered as 'solutions' or answers to any question need to be stored in a matrix.

Step 3: The best solution needs to be found by using a network analysis search algorithm to compare alternative solutions with criteria defining the optimum solution.

Over the last 30 years, network analyses have been applied to geotechnical situations.

Examples of relevance to the present technical paper include location of the critical minimum shear strength path through a rock mass with one set of defects (Glynn et al., 1978) and estimation of the displacementdependent shear strength developed along defects, with rough undulating surfaces and user designated infill thickness, as the defect's upper surface displaces in various directions relative to the defect's matching bottom surface (Baczynski, 1986; Baczynski et al., 1986)

\subsection{General methodology}

The existing STEPSIM4 and the proposed STEPSIM4-REVISED (STEPSIM5) are significantly different methods for evaluating the shear strength of critical step-path traverses through rock mass slopes.

As shown in Figures 3 and 5, the STEPSIM4 method progressively generates a step-path traverse through the target slope. The traverse starts at the target slope toe. The generation process is iterated until the traverse attains its user-nominated length and exits the rock mass at some location behind the slope crest. Each traverse is simply a string of interconnected elements consisting of rock mass 'cells', defects, and bridges between defects. Geotechnical attributes of each element are statistically assigned. The STEPSIM4 analysis result is purely a function of conditions simulated along the traverse irrespective of whatever rock mass conditions may exist in the rest of slope.

In contrast to STEPSIM4, the proposed STEPSIM4-REVISED method requires the pre-definition of geotechnical conditions for the entire rock mass slope or, more strictly, at least for the rock mass 'corridor' through which the critical path ultimately traverses, before network analysis task commences. The concept of rock mass 'corridor' is important because the need to only assign geotechnical parameters to a corridor within the mass drastically reduces the overall computing effort required to set up the slope model and this approach is also consistent with the way that the rock mass network is actually searched in a step-wise manner to identify the minimum shear strength traverse.

For purposes of illustrating the network analysis methodology, the example presented below has assumed that geotechnical parameters are assigned to the entire slope. The slope is three-dimensional (3D). The described network analysis is performed on successive 2D vertical sections/slices through the 3D slope mass to arrive at a quasi three-dimensional solution.

The network analysis algorithm comprises several key steps.

Step 1: Define the physical target slope limits for the network analysis - i.e. 3D slope model and the number of $2 \mathrm{D}$ vertical sections through the slope to be assessed. Commence model generation for the first section.

Step 2: As per conventional slope stability analyses, review the defect data and undertake a kinematic stability analysis to identify slope failure mode(s). If dominant slope failure mode involves sliding, then identify the shallower dipping defect sets that will be associated with shear sliding displacements and the steeper defect sets that will be associated with tensile separation (see Figures 6 and 7) at onset of slope instability. 
Step 3: Partition the first section into a regular array/network of equal-size 'micro-cells' (see Figure 8). In terms of network analysis terminology, each column of micro-cells is known as a 'stage' and each micro-cell in a stage is known as a 'state'. Network analysis 'accuracy' depends on micro-cell size; the smaller the cell, the more accurate the final solution (i.e. a concept akin to pixel size in digital cameras).

Step 4: Statistically assign intact rock properties (unconfined compressive strength, tensile strength, friction angle and cohesion) to each micro-cell (see Figure 8). Retain copy of this data array.

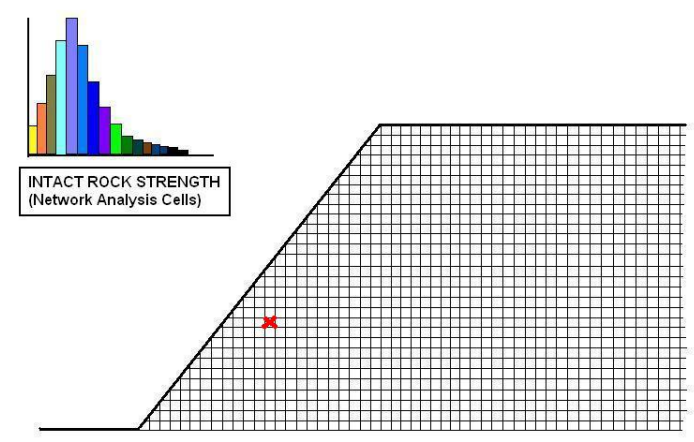

Figure 8 Slope partitioned into regular array micro-cells (i.e. 'stages' and 'states')

Step 5: Aggregate blocks of micro-cells into rock mass 'mega' cells. Statistically assign rock mass properties (unconfined compressive strength, tensile strength, friction angle and cohesion) to each mega-cell (see Figure 9). Retain copy of this data array.

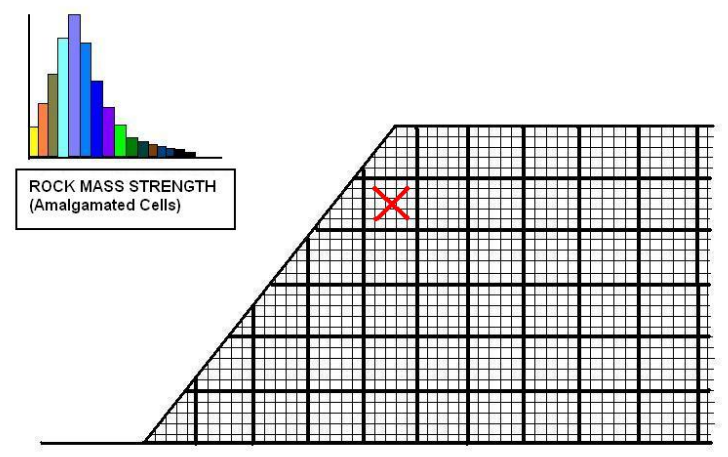

\section{Figure 9 Micro-cells aggregated into rock mass mega-cells}

Step 6: Either import existing digital data or statistically generate digital data for each set of defects within the target slope. Existing data may be available from SIROVISION photography of slopes. Statistically generated data may comprise computer simulated defect patterns as described in Baczynski (1980) and other authors. Sort defect data by X, Y, Z coordinates to identify the subset of defects whose traces occur on the first section through the target slope. Superimpose defect traces (see Figure 10) over a copy of the array for micro-cells generated in Step 4 for intact rock properties and assign statistical defect properties (shear strength, tensile strength) to those micro-cells intersected by the respective defects. In situations where several defects traverse a specific micro-cell, retain in memory the defect with the minimum strength and failure mode condition. Iteratively superimpose the trace of each defect until all defects are processed. Retain copy of this data array. Maintain a duplicate data array that tracks defect situations such as defect cross overs/intersections and so on.

Step 7: Commence systematic network analysis through the array of micro-cells (stages and states). Start at the toe of the slope (i.e. coinciding with the Stage 1, State 6 micro-cell in Figure 12 Plot A) and iteratively continue the network analysis search to identify the minimum shear-strength step-path traverse through the target slope. 


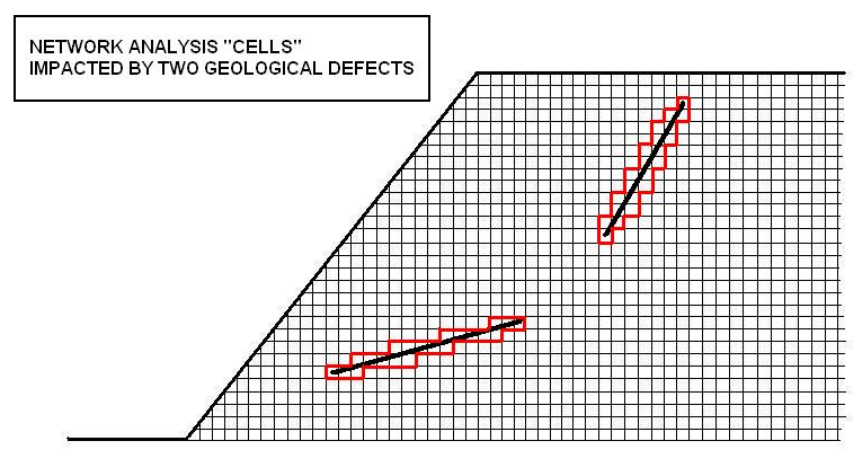

Figure 10 Superimpose defects on slope array of micro-cells

The network analysis search algorithm for a subset of a target slope is schematically illustrated in Figures 11 and 12 (Plots A to L).

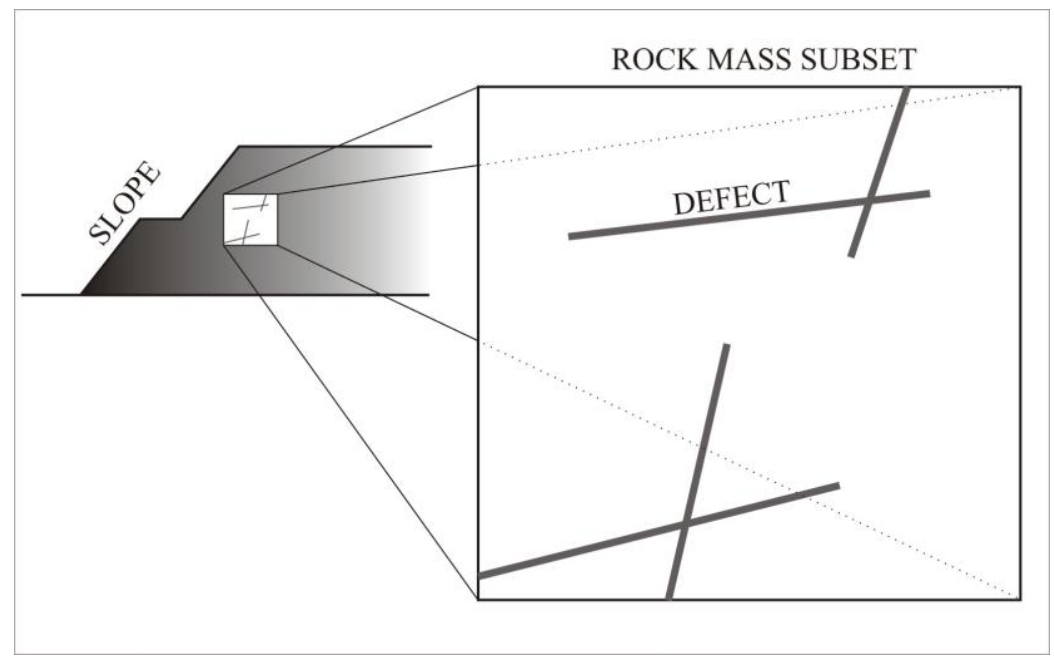

Figure 11 Rock mass subset of a conceptual slope

Figure 11 shows an example of a target slope with a rock mass subset 'window' (mega-cell) containing four defects (one set shallow dipping, the other set steep). Figure 12 (Plots A to L) illustrates the iterative steps in the network analysis procedure. For conceptual purposes, the following shear and/or tensile dimensionless strengths were assigned to represent various ground conditions in the shown micro-cells (i.e. states).

Intact rock shear strength $=20$

Defect sliding strength $=7$
Intact rock tensile strength $=10$

Defect tensile strength $=4$
Defect junctions/cross overs $=2$

In a STEPSIM4-REVISED analysis for a real slope, conditions in each micro-cell/state would be statistically defined, with the range of assigned shear and tensile strengths reflecting the variability in actual conditions in the real slope. Statistical variability in conditions would be derived by detailed structural mapping of actual slope faces, laboratory testing of intact rock and defects and by using available techniques such as the HoekBrown equations for estimating the strength of jointed rock masses (as applied in Baczynski, 1980, Little et al., 1997-2000 and Baczynski et al., 2001). Invariably, the process is partially interpretative and judgemental. Accordingly, the analysis will be as valid as the field and laboratory data that has been collected or estimated and as the current understanding of rock mass strength and behaviour will allow.

The following steps are involved in the proposed network analysis procedure.

Step A: a micro-cell array is superimposed over the rock mass subset illustrated in Figure 11. This array comprises Stages 1 to 6 and each stage has six states (Figure 12, Plot A). 
Step B: On the basis of defect dips, shear sliding failure is attributed to the flatter defects and tensile failure to the steeper defects. As per Step 7, a numerical strength is assigned to each micro-cell/state in the array; the respective strength values are shown in the top left-hand corner of each micro-cell (Figure 12, Plot B).

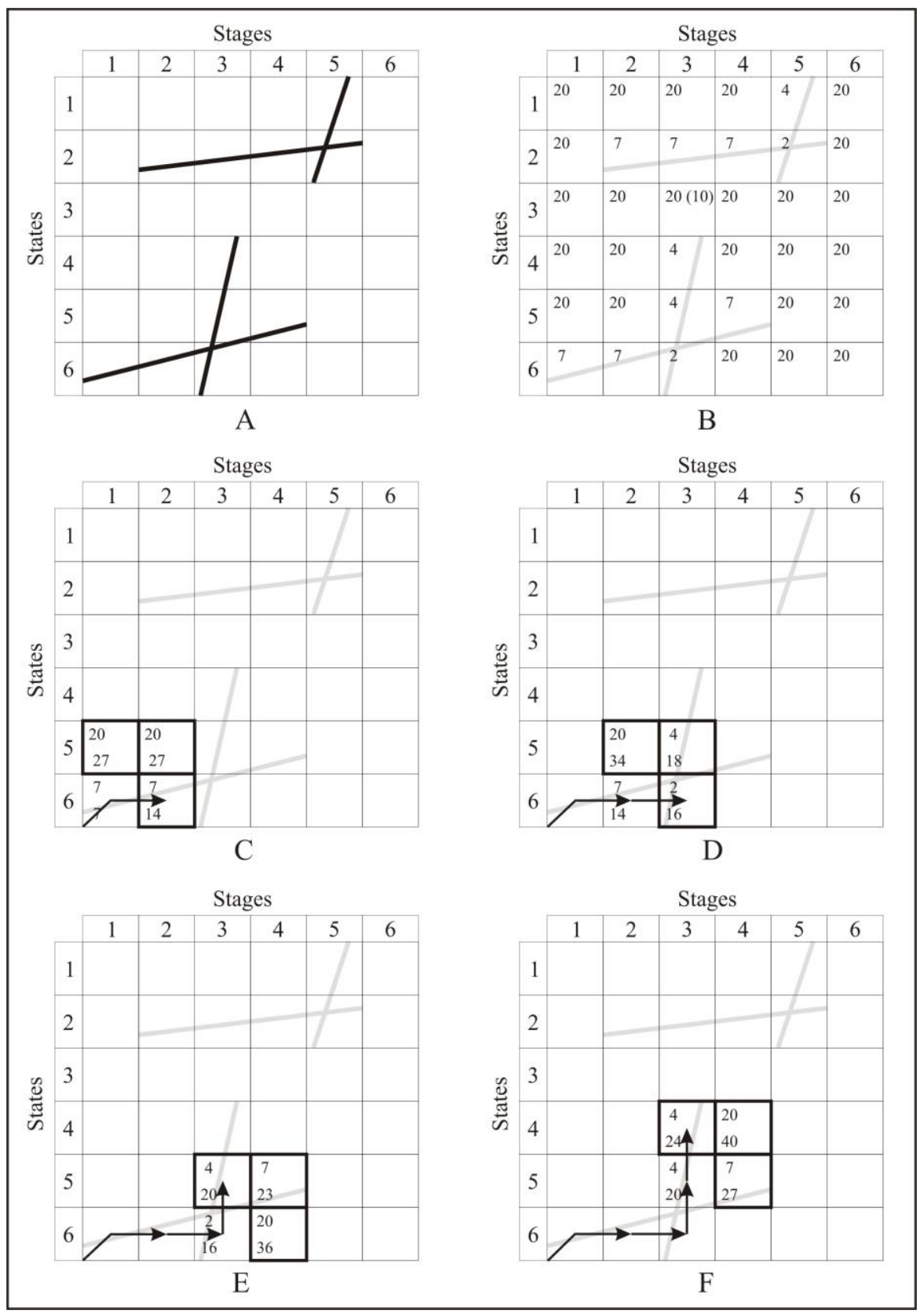

Figure 12 (A-F) Network analysis applied to rock mass subset of a conceptual slope

Step C: The network analysis step-path traverse starts at the bottom left-hand corner of the stage-state array (i.e. micro-cell at Stage 1, State 6 location). Analysis will progress from left to right across the array. The traverse will end somewhere along either the top or the right-hand side perimeter of this array. Three conditions have been imposed for progressing from one stage to the next. These conditions are that, from a current position, the traverse can only extend to (1) a cell immediately above, (2) a cell above and diagonally across on the right-hand side or (3) a cell horizontally adjacent on the right-hand side. The traverse cannot step downwards or back into an earlier entered stage of the array (Figure 12, Plot C).

The cumulative strength (shearing resistance) required to progress the traverse from its current position (current state in the current stage) to the next allowable state (micro-cell) is assigned to the three allowable adjacent micro-cells; the respective cumulative values are shown in the bottom left-hand corner of each cell. 
In the shown example (Figure 12, Plot C), the cumulative strengths are 27, 27 and 14 for the states vertically upwards, diagonally upwards and horizontally across states/cells, respectively. The traverse is extended in the direction of the adjacent state (cell) with the least cumulative strength. In this case, the cell is horizontally adjacent to the starting cell.

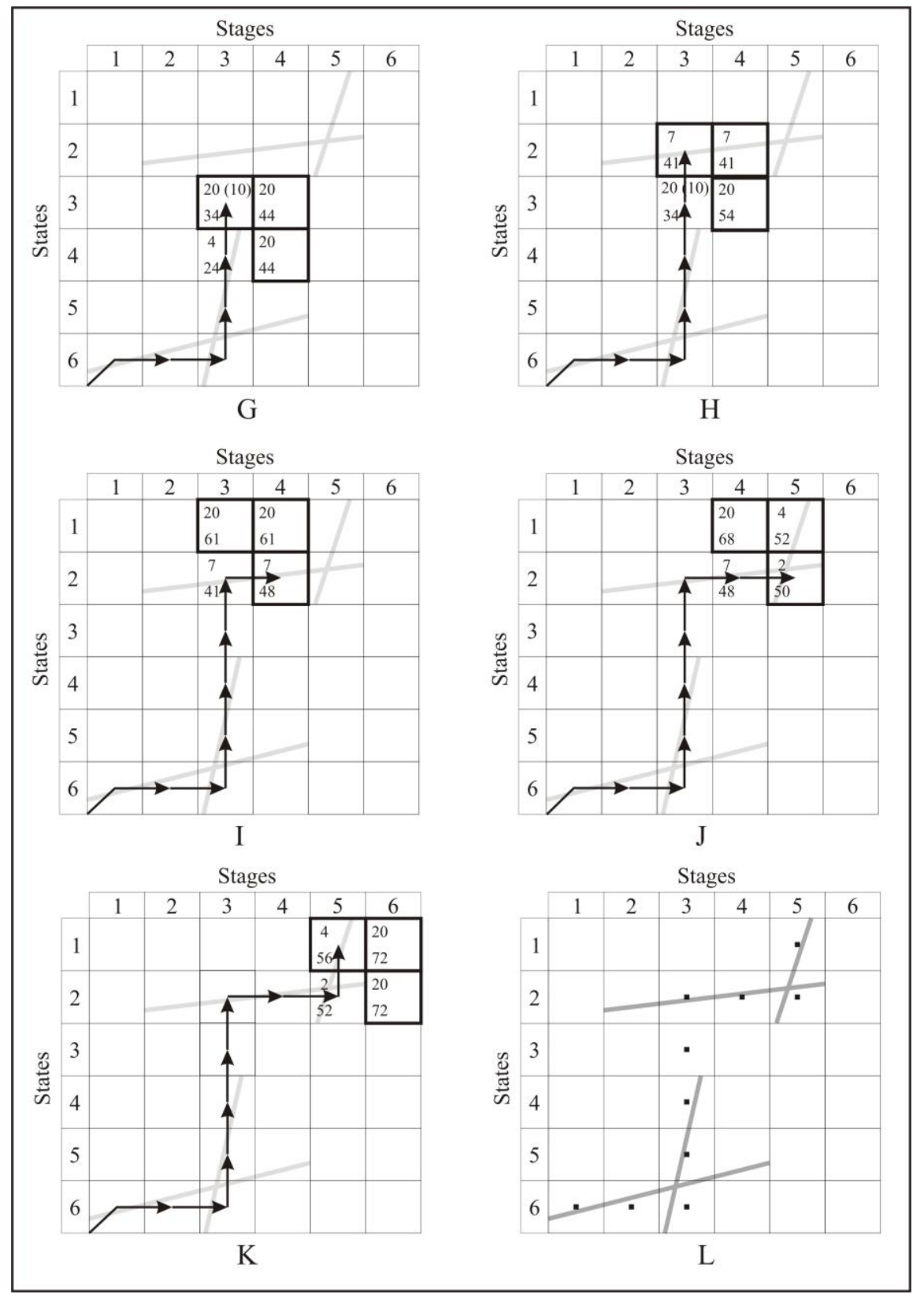

Figure 12 (G-L) Network analysis applied to rock mass subset of conceptual slope

Step D: The process described above is iterated through successive micro-cell/stages-states until the minimum strength traverse exits the network array at a cell along the top or right-hand side perimeter. In this case, the exit micro-cell is located at the Stage 5, State 1 position (Figure 12, Plots D to K).

Figure 12 (Plot L) uses large dots within the micro-cells to flag the path of the network analysis computed minimum strength traverse. This example demonstrates that the network analysis has successfully located the traverse that a person may have intuitively interpreted through the illustrated jointed rock mass. This analysis has numerically achieved results similar to those visually achieved in Figure 7. Whenever the results of an analysis do not meet expectations, then two issues need close re-examination: the input assumptions and personal beliefs/bias of likely failure mode. 
In a more complex model representing actual slope conditions with micro-cells statistically assigned strength parameters, some defects may be simulated as being statistically stronger than some 'soil-like' rock cells. In these extreme circumstances, perhaps the network analysis determined traverse may not necessarily always coincide with a path that maximises the use of defects, especially those defects failing by tensile separation, along the traverse.

\subsection{Network analysis precedence and decision points}

The presented example has highlighted two conditions that are inherent in any network analysis.

- Order of precedence.

- Decision points.

With respect to order of precedence, the minimum shear strength traverse is achieved when the network analysis search algorithm maximises the cumulative length of the weakest elements along the step-path traverse. In the provided example, the maximisation process has the following order of precedence.

Defects tensile $>$ Defect cross-over $>$ Defects shear $>$ Rock Mass tensile $>$ Rock Mass shear $>$ Intact Rock tensile > Intact rock shear

In Figure 12 (Plot G), the search algorithm had also reached a decision point in cell (3, 4); i.e. (Stage, State). This cell is surrounded by intact rock elements in all allowable directions of traverse advance. The choice made was to step vertically upwards. This choice occurred because the writer designated 10 and 20 units for the tensile and shear strength of intact rock, respectively. Where such decisions arise for a large number of successive cells, the traverse path choice may not necessarily be apparent because the search algorithm has no prior knowledge of where the longest defects are located ahead of the traverse. The risk is that the minimum strength path may not necessarily be identified. This issue may perhaps be resolved by multi-pass search algorithm. Large-size cells (say $1 \mathrm{x} 1 \mathrm{~m}$ ) could be initially used, with this search locating the longest defects and providing general direction for advance of the step-path traverse through the target slope. Cell sizes could be successively reduced (say, to $0.3 \times 0.3 \mathrm{~m}$; and ultimately to $0.05 \times 0.05 \mathrm{~m}$ ) to refine the traverse through those parts of the matrix that comprise rock mass and intact rock elements.

\section{Conclusions}

This paper reviews the basis, assumptions and limitations of the existing STEPSIM4 method and proposes a new network analysis based STEPSIM4-REVISED approach to identify the physical location and to assess the cumulative strength mobilised along critical step-path traverses through actual or computer simulated jointed rock mass slopes. Conceptually, the STEPSIM4 principles are simple and represent the current level of technology for 2D computer simulation and assessment of the statistical strength likely to develop along critical step-paths in rock mass slopes. However, the existing STEPSIM4 approach has a number of disadvantages. These include:

- The current analysis is solely 2D and cannot be extended into 3D.

- The average dip of a traverse and direction of sliding within the rock mass coincide with the straight line that connects the start of the traverse at the slope toe with the end of the traverse at some location behind the slope crest, this simplification is rarely the case in actual rock masses.

- Sliding type failure mode occurs on all defects, irrespective of mean set orientations; this failure mode is an oversimplification that disregards the tensile separation/opening that is often observed on members of steeply dipping defect sets in actual rock masses during initial stages of failure.

- Entire defect lengths are used to define each segment along a traverse; definition of traverse segments by using only part length of individual defects is not possible.

Conceptually, the proposed STEPSIM4-REVISED network analysis principles are more complex, a 3D defect model is required for the target rock mass and the analysis is much more demanding in terms of the computing effort required to setup the slope model and to numerically identify the critical path. After the STEPSIM-REVISED (i.e. STEPSIM5) software code has been developed, the associated network analyses 
will require access to high speed personal computers (PC) with significant RAM and hard drive memory capability.

However, notwithstanding the above comments, the writer believes that the STEPSIM4-REVISED method will successfully address all of the STEPSIM4 disadvantages; with the proviso that a 3D capability will be quasi-achieved by generating and assessing closely spaced 2D sections through a 3D slope. There is also a measure of perhaps conceptual elegance and numerical sophistication in the network analysis STEPSIM4REVISED method when compared to the existing STEPSIM4 approach. However, the practical application and benefits of proposed network analysis technique still need to be demonstrated.

A key issue in any simulation process is an adequate understanding of the real rock mass slope conditions. If this understanding is poor or just very approximately estimated, then no amount of sophisticated computer simulation effort will improve the accuracy of the shear strength estimate.

At this stage, it is unknown and speculative whether the STEPSIM4-REVISED results will be significantly different from those that would be computed via the existing STEPSIM4 software. The writer suspects that the network analysis technique will yield somewhat reduced shear strength values for critical step-path traverses through rock mass slopes than would have been otherwise estimated by STEPSIM4; but this suspicion needs to be proven and is part of the process of understanding limitations that all models have. If the limitations are understood, then a result that does not appear to be right must be critically examined.

\section{Acknowledgements}

The initial STPSIM step-path software was conceptualised and developed by Dr Barry McMahon in 1979 during his pit slope design work at the Bougainville open pit mine in PNG. Without Dr McMahon's initial software, it is unlikely that STEPSIM4 would have eventuated in 1997. Ok Tedi Mining Limited (OTML) supported the development of STEPSIM4 as part of their 1997-2000 pit slope design optimisation study. OTML also funded the initial conceptual work for the STEPSIM4-REVISED software (Baczynski, 2002). Development of the proposed STEPSIM5 software is being sponsored by the CSIRO under their Large Open Pit project and by OTML. Karl Smith's and Daniel Hastings' editing assistance in preparing this paper are greatly appreciated.

\section{References}

Baczynski, N.R.P. (1980) Rock Mass Characterization, PhD Thesis, Department Mining and Metallurgy, University of Melbourne, dated Nov 1980 (awarded 1981), 233 p.

Baczynski, N.R.P. (1986) SSNAP3D: Shearing Response along Rough Fractures: Part 1 - Theoretical Considerations, Bull. Assoc. Engng. Geologists, Vol. XXIII, No. 2, pp. 167-179.

Baczynski, N.R.P., Willoughby, D.R. and McBurney, C.C. (1986) SSNAP3D: Shearing Response along Rough Fractures: Part 2 - Field Application, Bull. Assoc. Engng. Geologists, Vol. XXIII, No. 2, pp. 181-196.

Baczynski, N.R.P. (2000) STEPSIM4 'Step-Path' Method for Slope Risks, GeoEng2000, Int. Conf. Geotech. \& Geol. Engng., Melbourne, Victoria, Australia, 19-24 November.

Baczynski, N., Marples, R., Tamplin, S. and Choros, E. (2001) Application of slope stability risk design process to open cut mines, Proceedings Symposium on Geological Hazards: The Impact on Coal Mining, Lake Macquarie, 15-16 November, pp. 151-171.

Baczynski, N.R.P. (2002) Upgraded STEPSIM4: Use of network analysis techniques for the determination of shear strength along critical step-paths through rock mass slopes. Internal Report prepared for OTML, 28 Dec, 21 p., Figures 1 to 26, Appendices A to C.

Brandes, U. and Erlebach, T. (2005) Network Analysis, Methodological Foundations, LNCS 3418 Tutorial, Springer Verlag, Berlin Heidelberg, 471 p.

Glynn, E.F., Einstein, H.H. and Veneziano, D. (1978) The probabilistic model for shearing resistance of jointed rock. Proceedings 19th US Symp. Rock Mech., Stateline, Nevada, pp. 66-76.

Little, T.N., Cortes, J.P. and Baczynski, N.R. (1997-2000) Risk-based slope design optimisation study for the Ok Tedi copper-gold mine. Volumes 1 to 8, and Executive Summary, Ok Tedi Mining Limited, Mine Technical Services Department, Geotechnical Engineering Section. 